\title{
Multi-scale features extraction from baseline structure MRI for MCI patient classification and $\mathrm{AD}$ early diagnosis
}

\author{
Kun $\mathrm{Hu}^{\mathrm{a}}$, Yijue Wang ${ }^{\mathrm{a}}$, Kewei Chen ${ }^{\mathrm{c}}$, Likun Hou ${ }^{\mathrm{b}}$, Xiaoqun Zhang ${ }^{\mathrm{b}, \mathrm{a}, *}$ \\ ${ }^{a}$ Department of Mathematics, Shanghai Jiao Tong University, Shanghai, China \\ ${ }^{b}$ Institute of Natural Sciences, Shanghai Jiao Tong University, Shanghai China \\ ${ }^{c}$ Banner Alzheimer's Institute and Banner Good Samaritan PET Center, Phoenix, Arizona, U.S.A.
}

\begin{abstract}
In this study, we investigate multi-scale features extracted from baseline structural magnetic resonance imaging (MRI) for classifying patients with mild cognitive impairment (MCI), who have either converted or not converted to Alzheimer's disease (AD) three years after their baseline visit. Total 549 subjects from the Alzheimer's disease Neuroimaging Initiative (ADNI) database are included, and there are 228 Normal controls (NC), 133 MCI patients (71 of them converted to AD within 3 years, referred as MCI converters, or MCIc) and 188 AD patients. The images are preprocessed with the standard voxel-based morphometry method with segmentation of grey matter, white matter and cerebrospinal fluid. Wavelet frame, a multi-scale image representation approach, is applied to extract features of different scales and directions from the processed grey matter image data. The features are extracted for both whole grey matter images and grey matter images of the hippocampus region. The support vector machine is adopted to construct classifiers for MCIc and MCI non-converters (MCInc). The accuracy using a leave-one-out procedure for classification of AD vs NC and MCIc vs MCInc is $84.13 \%$ and $76.69 \%$ respectively, both achieved by local hippocampus data. Our study shows that the proposed multi-scale method is capable of discriminating MCI converters and non-converters, and it can be potentially useful for MCI prognosis in clinical applications.
\end{abstract}

Keywords: Alzheimer's disease; MCI; structural MRI; tight wavelet frame; multi-scale, SVM

\section{Introduction}

Alzheimer's disease (AD) is a chronic neurodegenerative disease which is characterized by an initially gradual and then accelerated decline of cognitive function, and it accounts for more than half of dementia patients who cannot live alone and thus need dedicated and often long-term care. The World Alzheimer Report [40] [30] indicates that the annual cost of the dementia care for the 35.6 million sufferers is around $1 \%$ of the global GDP and the number of sufferers is estimated to double in the next two decades. Meanwhile, there is no effective treatment to relieve AD, let alone a cure. Mild cognitive impairment

\footnotetext{
${ }^{*}$ Corresponding author

Email address: xqzhang@sjtu.edu.cn (Xiaoqun Zhang)
} 
(MCI) is a prodromal stage of $\mathrm{AD}$, and approximately $10 \%$ to $15 \%$ of individuals with MCI progress to AD per year [19]. At this relatively early stage, it is crucial to estimate the likelihood of conversion to $\mathrm{AD}$ as accurately as possible. With the development and advancement of neuroimaging techniques, there are discussions on the use of features based on such as magnetic resonance imaging (MRI) [39] [15] [1], fluorodexyglucose positron emission tomography (FDG-PET) [7] [18] to estimate the convertion rate. Evidence shows that these features do provide valuable information assisting the diagnosis or prognosis with increased sensitivity and accuracy, especially in the early stages.

Compared to PET techniques, MRI is relatively inexpensive and yet performs comparably; based on numerous studies. In this study, we aim to examine the feasibility of baseline MRI to differentiate stable and convertible MCI patients using a multi-scale method that, to the best of our knowledge, is a new method for analyzing MRI data and especially in the context of $\mathrm{AD}$ neuroimaging studies. We first apply this multi-scale technique to extract features from preprocessed grey matter images. Then we consider two ways, namely the global approach and the hippocampus approach, to construct classifiers to distinguish MCI converters (MCIc) and MCI non-converters (MCInc) via linear support vector machine (SVM). The leave-oneout procedure is used to estimate the performance of the classifiers. Compared to various methods in the literature, the accuracy rate of our method for MCI patients is $71.43 \%$ for the global approach and $76.69 \%$ for the hippocampus approach. The p-value of the two sample t-test demonstrates that the features of hippocampus approach have significant difference between the MCInc and MCIc samples, in line with the fact that our hippocampus feature analysis achieves a superior classification accuracy.

\section{Materials and Methods}

Data used in this work are collected from the Alzheimer's Disease Neuroimaging Initiative (ADNI) database (adni.loni.usc.edu). As a $\$ 60$ million, 5-year public private partnership, the ADNI was launched by the National Institute on Aging (NIA), the National Institute of Biomedical Imaging and Bioengineering (NIBIB), the Food and Drug Administration (FDA), private pharmaceutical companies and non-profit organizations. The goal of ADNI is to determine sensitive and specific AD classifiers which act as a signal of early disease and an assessment of treatment effects [27] [37] [38]. ADNI has long been dedicated to the investigation into the shift of the imaging biomarkers, e.g. serial magnetic resonance imaging (MRI) or positron emission tomography (PET), combined with clinical, genetic and biochemical evaluation of subjects due to the disease gradually progressing from normal to Alzheimer's Disease, especially the MCI as the transitional stage and early AD.

\subsection{Subjects}

This study includes 228 Normal controls (NC) samples, 133 MCI patients and 188 AD patients from one of the Standardized MRI Data Sets [43]: ADNI_1 screening 1.5T for easier comparison with other methods. We include 228 of $229 \mathrm{NC}$ subjects by excluding one sample due to missing information. In MCI study, 133 samples with converted time information are investigated and among them 71 patients are converted to AD within 36 months. The demographic data of the datasets are listed in Table 1 and Table 2. In the two tables, we list the $p$ values of a two-sample t-test of APOE 4 and MMSE Total Score of the two groups. 
We can see that the two features APOE4 and MMSE both have significant differences for the $\mathrm{NC}$ vs $\mathrm{AD}$ and the MCIc vs MCInc group.

Table 1: Demographic data of NC vs. AD subjects

\begin{tabular}{cccc}
\hline Variable & NC N=228 & AD N=188 & p \\
\hline Sex M:F & $118: 110$ & $99: 88$ & - \\
Age & $76.0(5.0)$ & $75.4(7.5)$ & - \\
APOE4 & $0.29(0.5)$ & $0.9(0.7)$ & $<0.001$ \\
MMSE Total Score & $29.1(1.0)$ & $23.3(2.0)$ & $<0.001$ \\
\hline
\end{tabular}

Table 2: Demographic data of MCI subjects

\begin{tabular}{cccc}
\hline Variable & $\mathbf{n}$ MCI N=62 & c MCI N=71 & p \\
\hline Sex M:F & $44: 18$ & $45: 26$ & - \\
Age & $75.4(8.0)$ & $74.8(7.0)$ & - \\
APOE4 & $0.5(0.6)$ & $0.8(0.7)$ & 0.008 \\
MMSE Total Score & $27.7(1.6)$ & $26.8(1.6)$ & 0.003 \\
\hline
\end{tabular}

\subsection{MRI Data Preprocessing}

In this study, all the samples were obtained by a scanner with a standardized highresolution MRI protocol. The size of the raw $k$ - space data is $192 * 192 * 166$ and the size of each voxel is $1.25 * 1.25 * 1.2 \mathrm{~mm}^{3}$. For each slice along the third dimension, the image is reconstructed as $256 * 256$ with zero-filling. Details can be found on the ADNI website 1 .

It is necessary to preprocess structural MRI data before further analysis. The preprocessed steps are performed with a widely used software SPM 2 (Statistical Parametric Mapping). We adopt a standard preprocessing procedure for MRI data. First, the VBM8 toolbox 3 in SPM8 is used to normalize T1 images in template space and to segment them into three regions: grey matter (GM), white matter (WM) and cerebrospinal fluid (CSF). The segmentation algorithm involved in SPM8 is adaptive Maximum a Posterior (MAP) and Partial Volume Estimation (PVE) technique [4]. The high dimensional normalization in VBM8 adopted the registration algorithm Diffeomorphic Anatomical Registration Through Exponential Lie Algebra (DARTEL) [17]. Through all the image registration iterations and template creation, grey matter maps are normalized to their average templates and further to the Dartel template in Montreal Neurological Institute (MNI) space. Then we obtain a normalized brain image, and three segmented regions: grey matter, white matter and CSF for all subjects. For a local analysis, we use WFU Pick Atlas (http://www.nitrc.org/projects/wfu_pickatlas/) to generate region of interest (ROI) masks based on the Talairach Daemon (TD) database and other human atlases. In this study, we use the automated anatomical labeling (AAL) [35]

\footnotetext{
${ }^{1}$ http://adni.loni.usc.edu/methods/documents/ mri-protocols/

${ }^{2}$ http://www.fil.ion.ucl.ac.uk/spm

${ }^{3}$ http://dbm.neuro.uni-jena.de/vbm8
} 
atlas to extract the hippocampus region from the segmented grey matter images. Figure 1 shows the flow chart of the pre-processing step. We then perform feature extraction from two groups of images: the whole grey matter image and hippocampus region masked one.

\subsection{Wavelet Frame}

Wavelets have long time been an important tool for signal/image processing. Wavelet transforms [25, 16, 10] are able to extract feature in different scales from signals. For instance, Liu et al (2014) [24] used a geometric wavelet to authenticate paintings of Van Gogh and their method achieves an accuracy of 92.19\%. Hackmack et al (2012) [20] exploited dual tree wavelet to obtain features to diagnose multiple sclerosis patients, and achieved an accuracy rate of $95.12 \%$. Wavelet tight frames is a generalization of the standard orthogonal system, generated by translation and dilation of a wavelet function. In recent years, overcomplete wavelet frames are largely adopted in imaging applications due to their effectiveness in sparse representation and associated algorithms, see [11] for more theory and recent image applications. In this work, we are interested in exploring the multi-scale representation by wavelet frames of structural MRI data and analyzing the feasibilities of these features for AD and MCI patients classification.

Rigorously, the wavelet frame is a representation system in the image space defined in $\mathbb{R}^{d}$, which is often modelled as the function space $L_{2}\left(\mathbb{R}^{d}\right)$. For simplicity here, we present the definition and notation for $d=1$. Note that

$$
L_{2}(\mathbb{R})=\left\{f(x) \mid\|f\|_{L_{2}(\mathbb{R})}:=\left(\int_{\mathbb{R}}|f(x)|^{2} \mathrm{~d} x\right)^{\frac{1}{2}}<\infty\right\} .
$$

Given $\Psi:=\left\{\psi_{1}, \ldots, \psi_{r}\right\} \subset L_{2}(\mathbb{R})$ and $\psi_{l, j, k}:=2^{j / 2} \psi_{l}\left(2^{j} \cdot-k\right)$, we define the wavelet system

$$
X(\Psi):=\left\{\psi_{l, j, k}: 1 \leq l \leq r ; j, k \in \mathbb{Z}\right\},
$$

and the system $X(\Psi) \subset L_{2}(\mathbb{R})$ is called a tight wavelet frame of $L_{2}(\mathbb{R})$ if

$$
\|f\|_{L_{2}(\mathbb{R})}^{2}=\sum_{g \in X(\Psi)}|\langle f, g\rangle|^{2}
$$

Here we use the inner product $\langle\cdot, \cdot\rangle$ in $L_{2}(\mathbb{R})$. Parseval's identity is equivalent to $f=$ $\sum_{g \in X(\Psi)}\langle f, g\rangle g$, for all $f \in L_{2}(\mathbb{R})$. This definition of the tight frame is sometimes called the tight frame with frame bound one or Parseval frame in some literature. We can easily check that an orthonormal basis is a tight frame. In fact, $X(\Psi)$ is called an orthonormal wavelet basis if it forms an orthonormal basis of $L_{2}(\mathbb{R})$ and analogously $X(\Psi)$ is called a tight wavelet frame if it forms a tight frame of $L_{2}(\mathbb{R})$.

One fundamental question is to characterize the wavelet frame system $X(\Psi)$ through its generators to be a tight frame or even an orthonormal basis for $L_{2}(\mathbb{R})$. In [32] the authors proposed to use general refinable functions, and the concept of unitary extension principle (UEP), which makes the construction of wavelet frames of $L_{2}\left(\mathbb{R}^{d}\right)$ painless. In fact, compactly supported wavelets with finite supported masks $\Psi:=\left\{\psi_{1}, \ldots, \psi_{r}\right\} \subset L_{2}(\mathbb{R})$ can be constructed from a compactly supported refinable function $\phi$ and a refinement mask $h_{0}$. The function $h_{0}$ is often called the low pass filter, and the finitely supported sequences $h_{1}, \ldots, h_{r}$ are called the high pass filters of the system or wavelet masks. For example, the 


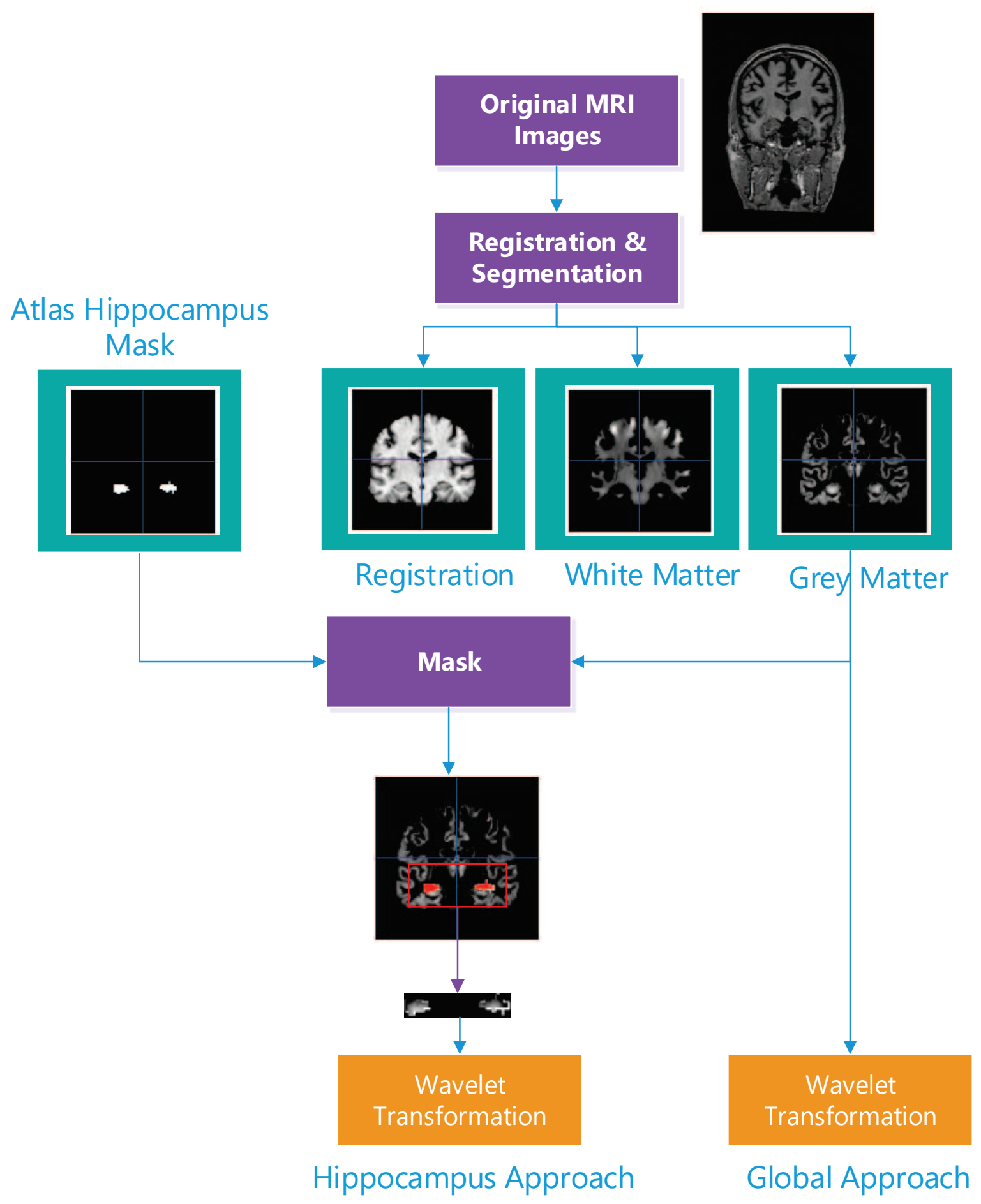

Figure 1: Flowchart of pre-processing step: After the registration and segmentation step, we obtain three images, one is the normalized image by transferring into standard brain space and the other two are the GM and the WM part of the normalized image, respectively. For further analysis, we are only interested in the GM image. We use the whole brain GM image for the global approach while the hippocampus part of the image is used for the hippocampus approach. 
piecewise linear spline tight frame introduced in [32] is widely used for representing many kinds of images, thanks to its sparse representation for most images with piecewise smooth structures. Its generating set consists of three piecewise linear spline functions associated with the following three finitely supported 1D discrete filters :

$$
h_{0}=\frac{1}{4}[1,2,1], \quad h_{1}=\frac{\sqrt{2}}{4}[1,0,-1], \quad h_{2}=\frac{1}{4}[-1,2,-1] .
$$

Among the three filters listed above, the first low pass filter can be used to extract the average intensity information of an image, and the other two high-pass ones are used to extract the boundary/edge information of 1D signals up to order 1 and order 2 . If multi-scale decomposition is applied, edge information of more orders can be extracted from 1D signals. In the application to $2 \mathrm{D}$ or $3 \mathrm{D}$ image data, $2 \mathrm{D} / 3 \mathrm{D}$ filters can be easily constructed from $1 \mathrm{D}$ ones by tensor products [12]. In the case of piecewise linear spline tight frame, when 3D tensor product is applied, the three associated filters will generate a set of $3 * 3 * 3=27$ discrete filters in 3D. Among all those 27 filters, there is one filter that is low-pass. In other words, there are 26 high-pass filters that are used for extracting boundary/edge information from images, which results in 26 channels of high-pass coefficients within each scale of decomposition. For different scales, dilation and zero filling can be applied to construct higher level filters. Hence the filter size at $j$-th scale is $\left(2^{j}+1\right) *\left(2^{j}+1\right) *\left(2^{j}+1\right)$. More details on discrete algorithms of wavelet frame transforms can be found in [11].

Figure 2 shows the filters at Scale I and Scale II for the purpose of illustration. The 26 channels of each scale are listed in row order. The size of the filters of Scale I and Scale II are $3 * 3 * 3$ and $5 * 5 * 5$ respectively. The colors of the cube represent the value of the 3D filter matrix according to the color bar. The larger this value is, the more the corresponding position counts in the extracted feature. We can observe that these filters extract information from MRI data in different directions and scales.

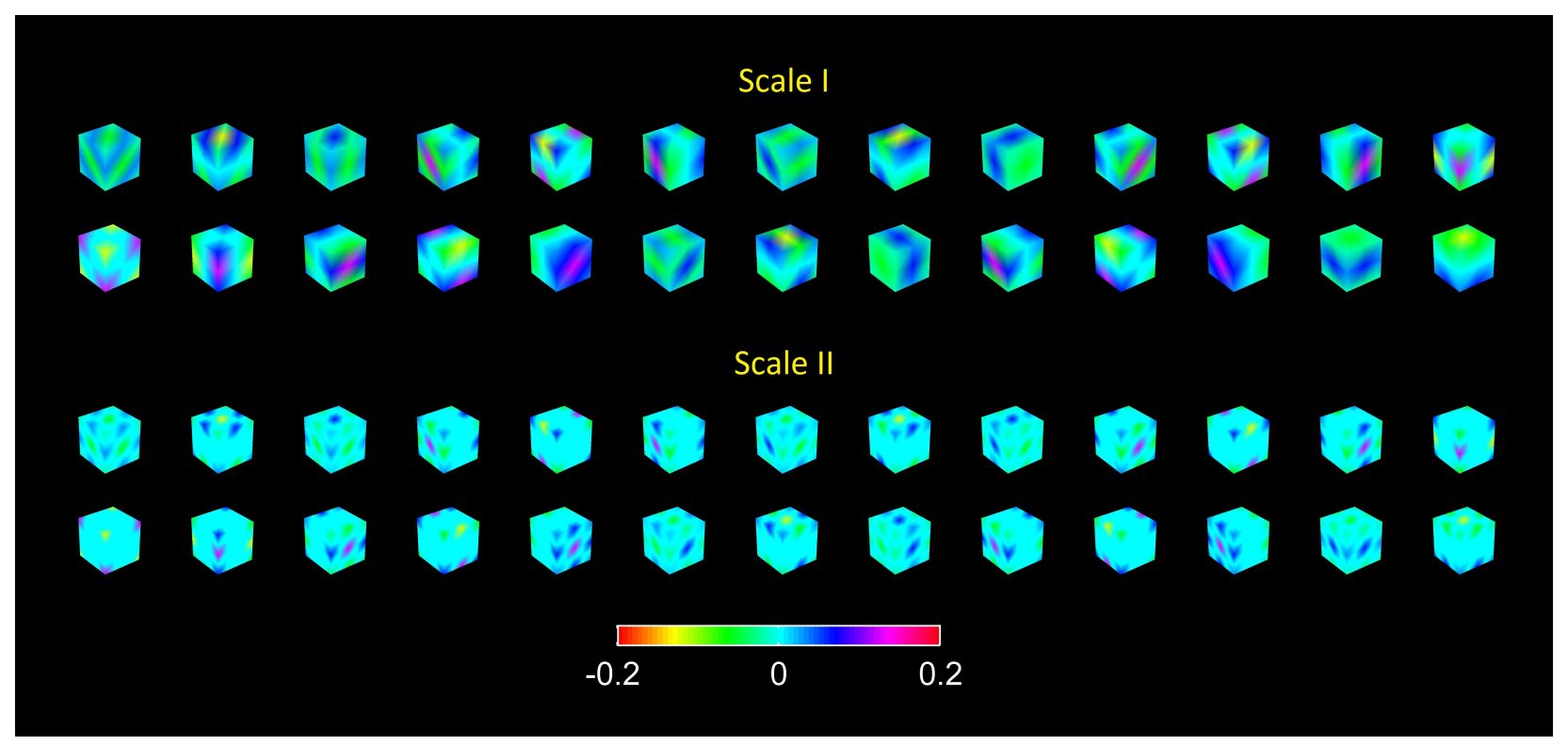

Figure 2: Wavelet frame filters of Scale I and Scale II (26 for each scale). These filters are used to extract different directional and edge information. 
In implementation of the wavelet transform, each image is decomposed into 5 or 6 scales depending on the size of the image. Each scale consists of 26 channels of high-pass coefficients and each channel is naturally a 3D matrix. Energy of these channels can be used as features to predict the conversion of the disease. We choose the second raw moment as the feature of each channel. Many statistics can be used as features, such as the sum of absolute value, second raw moment, skewness, mean, variance etc [24]. In this study, we choose the second raw moment since it gives the best performance when we test the different statistics on the trainning data. Therefore, the feature vector of the $i$-th subject at scale $j$ is denoted as:

$$
x_{i}^{j}=\left\{x_{i, 1}^{j}, \ldots, x_{i, 26}^{j}\right\}
$$

where $j$ stands for the scale and $j=1, \cdots 6$ for the whole GM image and $j=1, \cdots, 5$ for hippocampus GM sub-image. In total, there are $26 * 6=156$ features for the whole GM images and $26 * 5=130$ for hippocampus GM sub-images.

To provide an intuition of the coefficients of the tight frame, Figures 3 and 4 show a visualization of the wavelet frame coefficients of one MRI image. Figure 3 shows the eight channels in Scale I of which the variance of intensity are relatively larger. The isosurface and their corresponding values are shown in different colors. In different channels, wavelet frame introduces different directions of singularities, thus they contain information of the orientations of the edges of the original image. Figure 4 shows the coefficients of a fixed channel at different scales; as we can see, Scale I contains more detailed information than Scale II, Scale II is more than Scale III, etc. To further demonstrate the difference of each channel, histograms of the values of channels of Scale I are shown in Figure 5. Although values of these channels are around zero, their distribution is different in each channel. For instance, the histogram of Channel 1 and Channel 8 is overtly different; in Channel 1 there is no value around 0.5 or -0.5 . In brief, the wavelet frame transform provides information of the original image at different scales and directions, and it is able to extract sufficiently distinct features, which is potentially beneficial for classification.

As mentioned above, we perform feature extraction from MRI data in two ways: wavelet frame features obtained from whole GM image (global approach) and hippocampus ROI masked GM sub-images (local approach). The first eight element of the features vector $u_{i}, i=1 \ldots 8$ for ADNI_1 dataset by the local approach are illustrated in Figure 6. The $(i, i)-$ th element of diagonal line in Figure 6 is the histogram of feature $u_{i}$ divided by $\mathrm{AD}$ and Normal groups, where the red stands for the AD group and the green stands for the NC group. We can see that the red histograms and green histograms are separated. The $(i, j)$ grid in Figure 6 displays the feature pair $\left(u_{i}, u_{j}\right)$ in a scatter plot. It can be seen that the two groups of subjects distribute in different regions, and AD group tends to locate on higher energy region compared to the NC group in these channels. Intuitively, the two groups can be separated by a linear function in each grid. This motivates us to adopt a support vector machine (SVM) with a linear kernel to separate the data. We note that although other more complex varieties of kernels can be adopted (for instance, the Gaussian kernel is successful for many applications), the distribution of our data suggest that the linear kernel is a better choice, which is also certified by our numerical results. In the next section, linear SVM classifiers are trained at different scales separately or in combination, and we will compare the performances of these classifiers. 


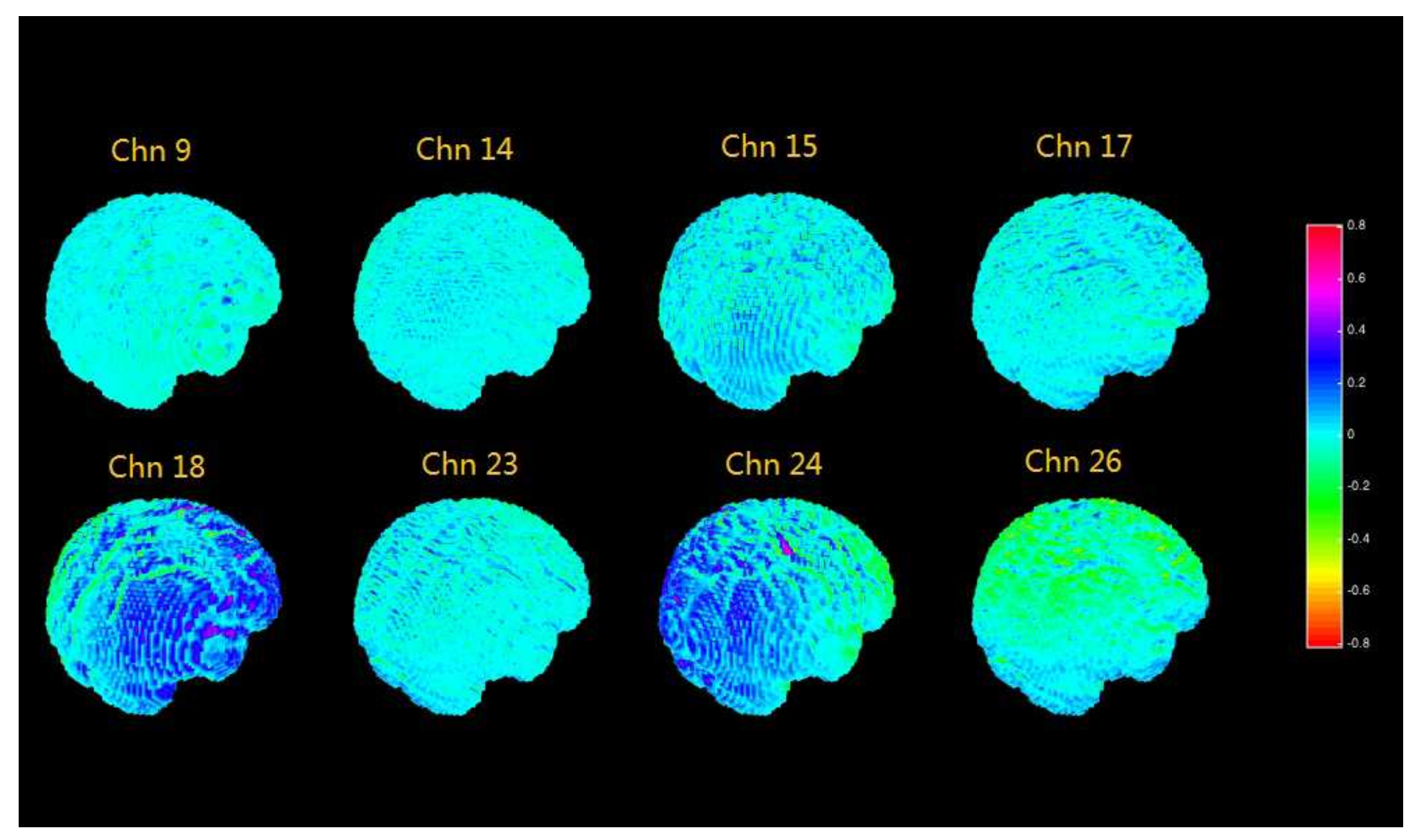

Figure 3: Eight exemplar high-pass channels at scale I of one subject are illustrated. We can observe directional edge information in these channels.

\subsection{Support Vector Machine}

Support vector machine (SVM) is a supervised learning method originally proposed by Vapnik et al. [8], and it has been widely used in supervised learning, especially for classification problems. With different kernel functions, SVM is flexible and suited for different types of features. In this work, a linear kernel function is adopted to fit a hyper-plane to separate high dimensional data points, as discussed in Section 2.3. A sequential minimal optimization algorithm [13] provided by MATLAB statistics toolbox is used to obtain SVM classifiers. Traditionally, SVM is used as a discrete binary classifier that gives 0 or 1 as the result. In this work, scores of SVM are adopted to help measure the distance of a feature vector to the decision boundary which ranges from $-\infty$ to $+\infty$ [29]. A positive score for a sample implies that this sample belongs to a pre-selected class, otherwise it is classified to the other class. Analogous to logistic regression, a posterior probability, which indicates a sample belonging to a particular class, can be computed by a function of scores of SVM. Hence the Receiver operating characteristic (ROC) curves of different SVM classifiers can be computed, and it is helpful to decide an appropriate threshold to the posterior probability in the context of the application [14] [31].

\section{Classification of $\mathrm{AD}$ and Normal}

In this section, various experiments of $\mathrm{AD}$ vs $\mathrm{NC}$ classification are performed to demonstrate the effectiveness of wavelet frames features. Both global and local approaches will be discussed in this section. Recall that for the global approach, the whole GM of the brain is 


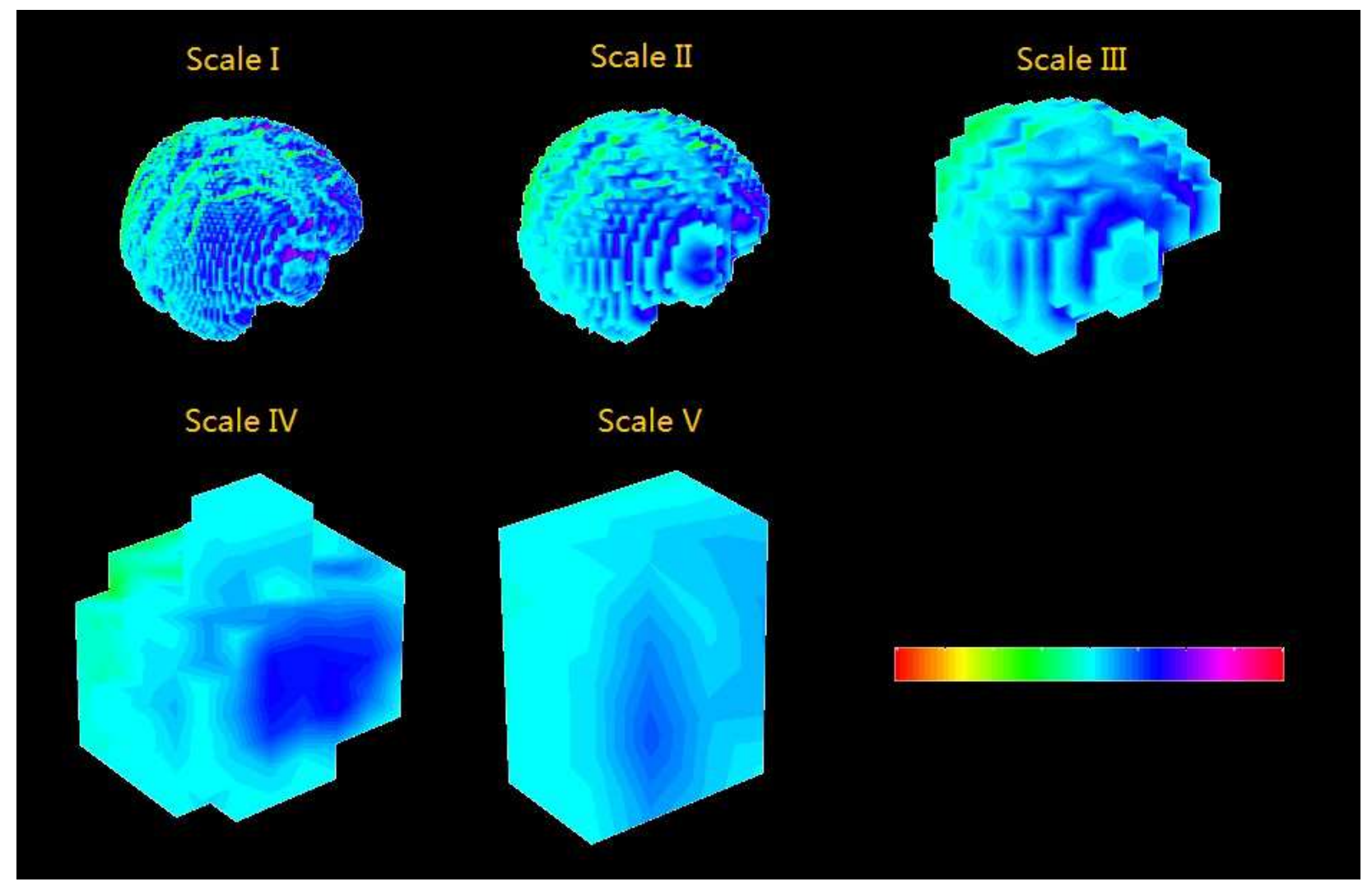

Figure 4: Isosurface of wavelet frame coefficients at five different scales of Channel 1 for one subject: More fine details are shown at the smaller scale. 

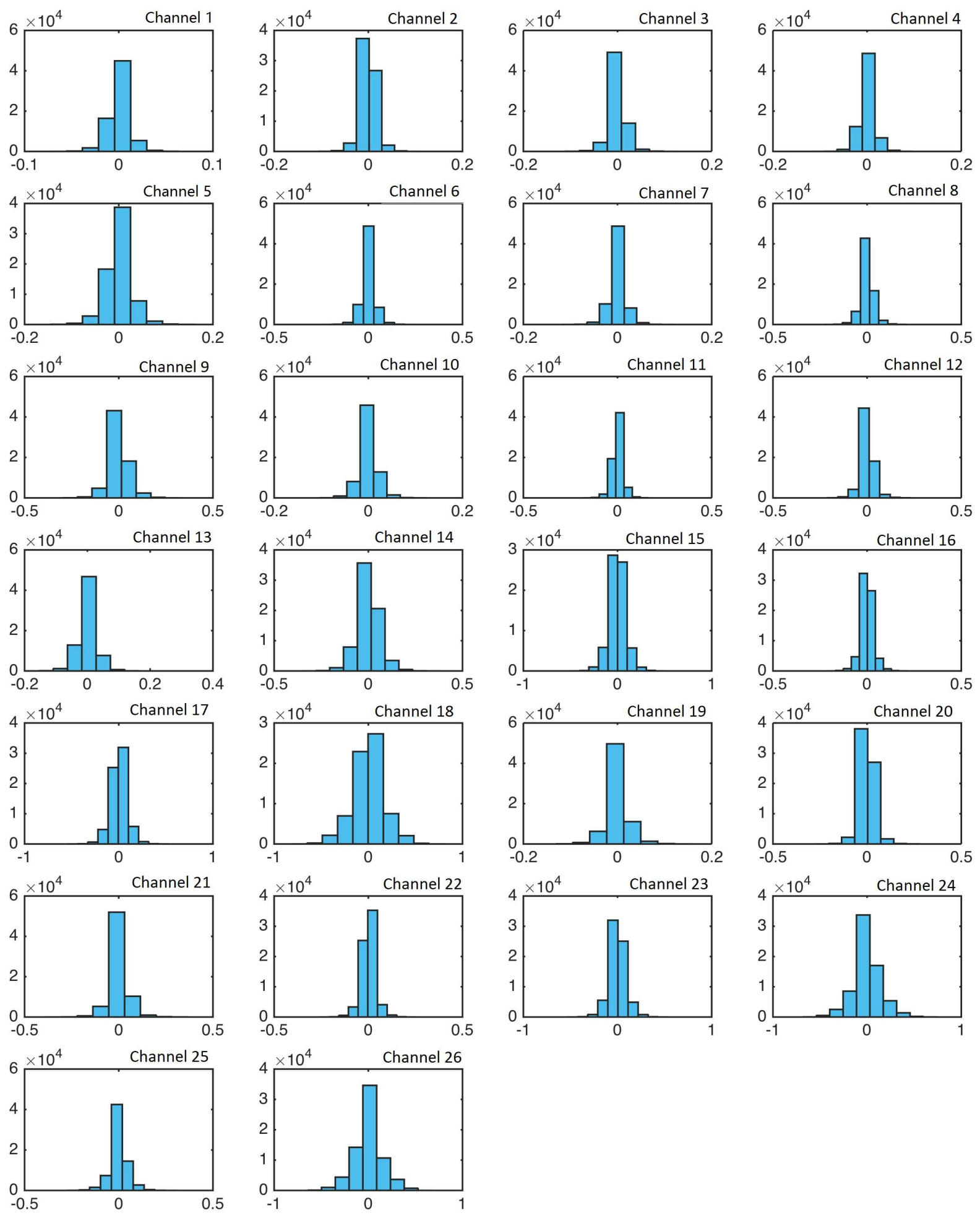

Figure 5: Histogram of each channel of Scale I of one subject: the distribution of intensity is different from channel to channel as different channels use different directional filters. 


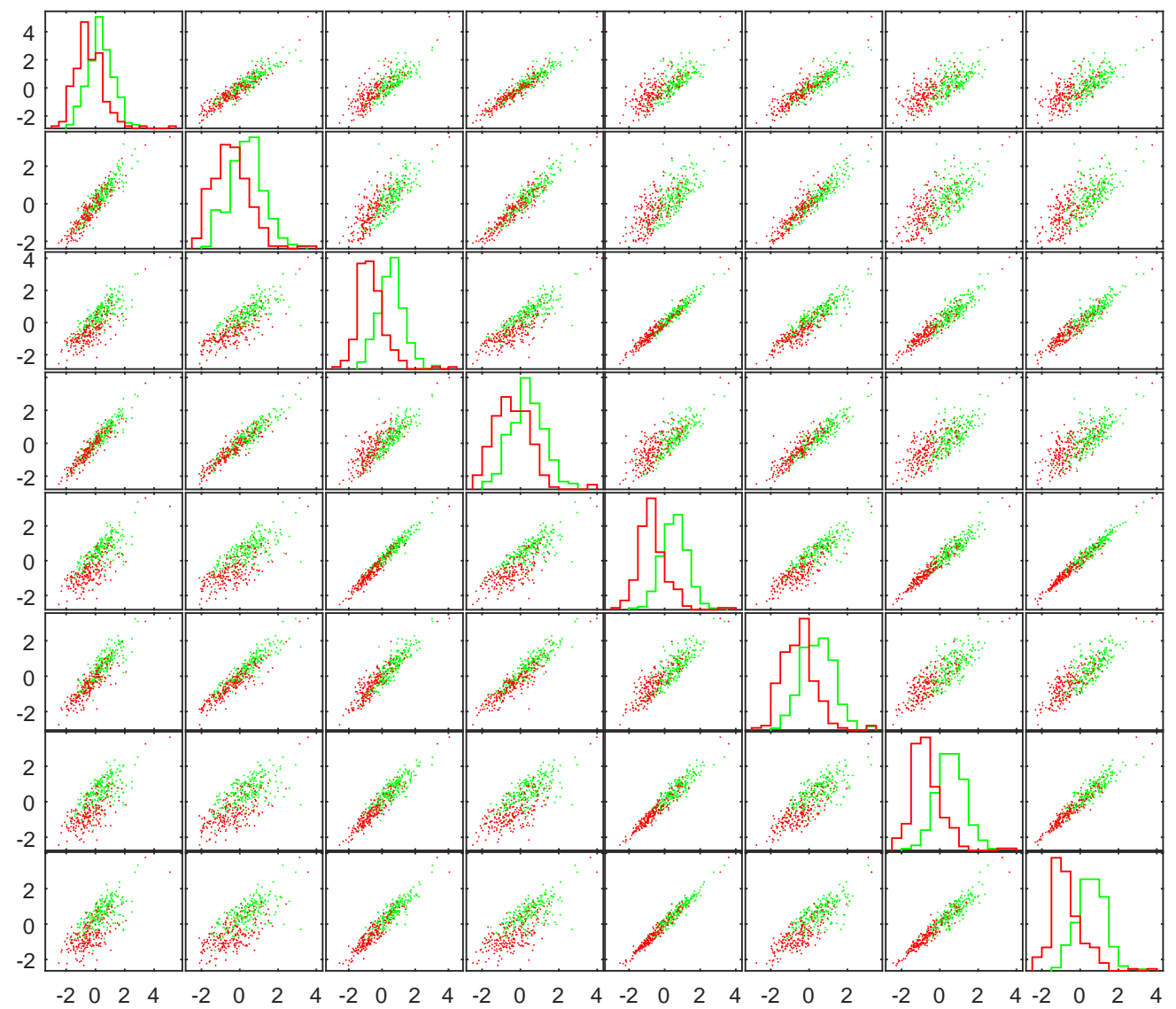

Figure 6: The scatter plot matrix of wavelet frame features of subjects in ADNI_1 dataset: the $(i, j)$ grid represents the distribution of feature pair $\left(x_{i}, x_{j}\right)$. The green points stand for NC subjects, while the red ones stand for $\mathrm{AD}$ subjects. The distribution of the subjects implies that a linear hyperplane can separate the two groups of data. 
used, while for the local approach, we only use the corresponding GM of the hippocampus ROI. In light of the methods for building these classifiers from AD and NC samples, we can find an intuition for the diagnosis of MCI patients in practice.

Normalization of wavelet frame features data is performed before we train a SVM classifier so as to avoid numerical difficulties, as recommended in [22]. A leave-one-out strategy is used to compute the classification accuracy, i.e., assuming the sample size is $n$, each time we use $n-1$ samples to train the SVM classifier, and the remaining one sample is used to test the classifier. Thus there are $n$ test results, and the accuracy is estimated by summing the correct ones and then dividing by $n$. The performance of these classifiers can be evaluated by accuracy, sensitivity and specificity:

$$
\begin{aligned}
\text { Accuracy } & =\frac{T P+T N}{T P+T N+F P+F N} \\
\text { Sensitivity } & =\frac{T P}{T P+F N} \\
\text { Specificity } & =\frac{T N}{T N+F P}
\end{aligned}
$$

where TP, TN, FP and FN stand for true positive, true negative, false positive and false negative, respectively.

\subsection{Global Approach}

By the method introduced in the last section, six scales with 26 channels each, totaling 156 features of each subject are computed for all the ADNI_1 screening 1.5T datasets subjects. The accuracy can be compared with other works which used the standardized data sets. Using different feature subsets, we trained several AD vs. Normal classifiers. The performance of the eight classifiers are listed in Table 3 . Figure 7 (a) shows the ROC curve of these classifiers. Table 3 shows that the best accuracy of $80.53 \%$ is achieved with Scale VI and its corresponding area under the curve (AUC) of Scale VI is 0.87. The other classifiers with different features also achieves high accuracy.

Table 3: Classification of AD vs. Normal Using Global Features

\begin{tabular}{cccccccccc}
\hline Features & Sensitivity & Specificity & $\begin{array}{c}\text { False } \\
\text { positive } \\
\text { rate }(\alpha)\end{array}$ & $\begin{array}{c}\text { False } \\
\text { negative } \\
\text { rate }(\beta)\end{array}$ & Power & $\begin{array}{c}\text { Likelihood } \\
\text { ratio } \\
\text { positive }\end{array}$ & $\begin{array}{c}\text { Likelihood } \\
\text { ratio } \\
\text { negative }\end{array}$ & Accuracy & AUC \\
\hline Scale I & $68.62 \%$ & $75.88 \%$ & $24.12 \%$ & $31.38 \%$ & 0.31 & 2.84 & 0.41 & $72.60 \%$ & 0.77 \\
Scale II & $69.68 \%$ & $73.68 \%$ & $26.32 \%$ & $30.32 \%$ & 0.3 & 2.65 & 0.41 & $71.88 \%$ & 0.81 \\
Scale III & $76.60 \%$ & $74.56 \%$ & $25.44 \%$ & $23.40 \%$ & 0.23 & 3.01 & 0.31 & $75.48 \%$ & 0.83 \\
Scale IV & $74.47 \%$ & $77.19 \%$ & $22.81 \%$ & $25.53 \%$ & 0.26 & 3.27 & 0.33 & $75.96 \%$ & 0.82 \\
Scale V & $76.06 \%$ & $82.89 \%$ & $17.11 \%$ & $23.94 \%$ & 0.24 & 4.45 & 0.29 & $79.81 \%$ & 0.85 \\
Scale VI & $76.60 \%$ & $83.77 \%$ & $16.23 \%$ & $23.40 \%$ & 0.23 & 4.72 & 0.28 & $80.53 \%$ & 0.87 \\
Scale I+II & $69.68 \%$ & $76.32 \%$ & $23.68 \%$ & $30.32 \%$ & 0.3 & 2.94 & 0.4 & $73.32 \%$ & 0.82 \\
Scale I-V & $73.40 \%$ & $76.32 \%$ & $23.68 \%$ & $26.60 \%$ & 0.27 & 3.1 & 0.35 & $75.00 \%$ & 0.85 \\
\hline
\end{tabular}

\subsection{Hippocampus Approach}

It has been confirmed that the hippocampus is a critical area of brain for learning and memory and is the first region to suffer damage in the Alzheimer's disease [28] [26]. Biomarkers 


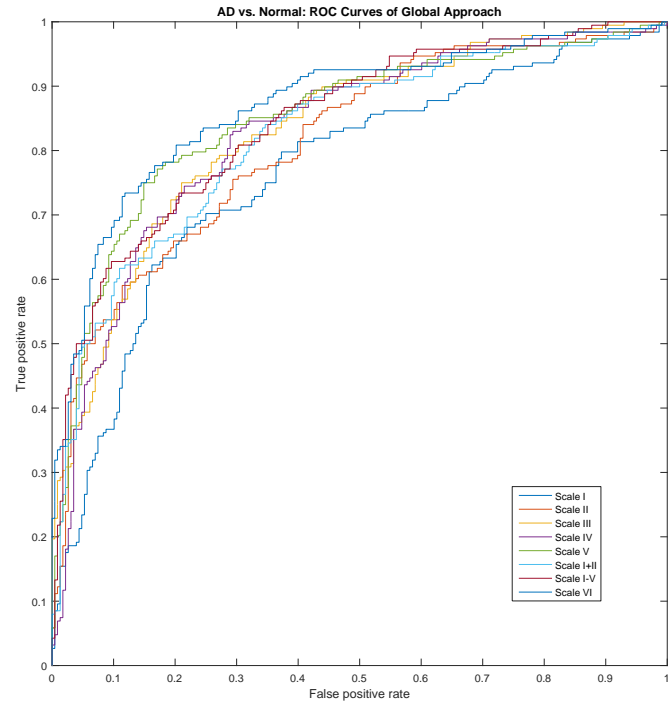

(a) Global Approach

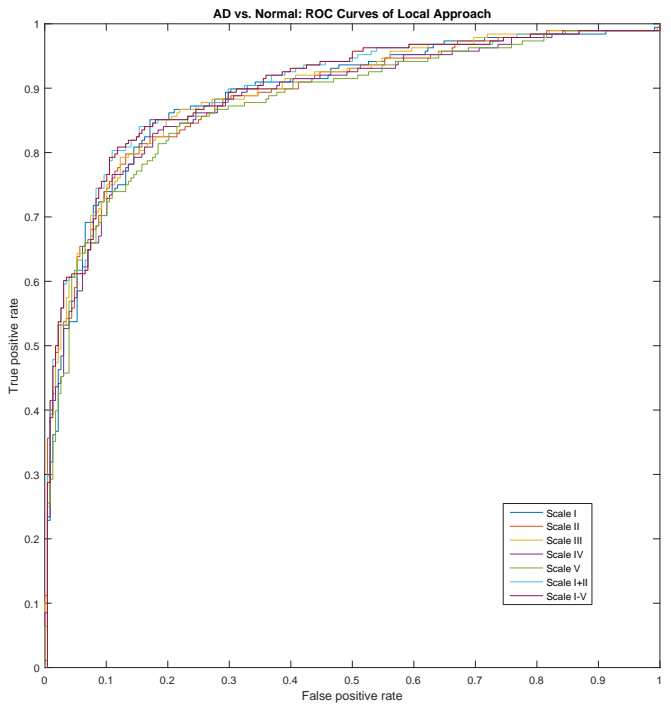

(b) Hippocampus Approach

Figure 7: ROC Curves of AD vs. NC Classifiers

of the hippocampus, especially volumetry have been used in AD diagnosing and fairly good results have been obtained [41] [6] [5]. For this reason, it is natural for us to pay special attention to the hippocampus area.

A cube of size $55 * 28 * 27$ (pixels) is used to extract the hippocampus region, both the left and right hippocampus, as described in Section 2.2. The wavelet transform is performed on this cubic region. The similar procedure of training SVM classifiers is applied. The performance of these classifiers is listed in Table 4.

Table 4: Classification of AD vs Normal using hippocampus features

\begin{tabular}{cccccccccc}
\hline Features & Sensitivity & Specificity & $\begin{array}{c}\text { False } \\
\text { positive } \\
\text { rate }(\alpha)\end{array}$ & $\begin{array}{c}\text { False } \\
\text { negative } \\
\text { rate }(\beta)\end{array}$ & Power & $\begin{array}{c}\text { Likelihood } \\
\text { ratio } \\
\text { positive }\end{array}$ & $\begin{array}{c}\text { Likelihood } \\
\text { ratio } \\
\text { negative }\end{array}$ & Accuracy & AUC \\
\hline Scale I & $80.85 \%$ & $83.77 \%$ & $16.23 \%$ & $19.15 \%$ & 0.19 & 4.98 & 0.23 & $82.45 \%$ & 0.89 \\
Scale II & $83.51 \%$ & $84.65 \%$ & $15.35 \%$ & $16.49 \%$ & 0.16 & 5.44 & 0.19 & $84.13 \%$ & 0.90 \\
Scale III & $80.85 \%$ & $82.02 \%$ & $17.98 \%$ & $19.15 \%$ & 0.19 & 4.5 & 0.23 & $81.49 \%$ & 0.89 \\
Scale IV & $84.57 \%$ & $85.53 \%$ & $14.47 \%$ & $15.43 \%$ & 0.15 & 5.84 & 0.18 & $85.10 \%$ & 0.91 \\
Scale V & $81.91 \%$ & $79.39 \%$ & $20.61 \%$ & $18.09 \%$ & 0.18 & 3.97 & 0.23 & $80.53 \%$ & 0.88 \\
Scale I-V & $80.32 \%$ & $84.65 \%$ & $15.35 \%$ & $19.68 \%$ & 0.2 & 5.23 & 0.23 & $82.69 \%$ & 0.89 \\
Scale I+II & $82.45 \%$ & $85.53 \%$ & $14.47 \%$ & $17.55 \%$ & 0.18 & 5.7 & 0.21 & $84.13 \%$ & 0.90 \\
\hline
\end{tabular}

The classifier with scale IV achieves the best accuracy of $85.10 \%$, sensitivity of $84.57 \%$, specificity of $85.53 \%$ and AUC of 0.91. Figure 7 (b) shows ROC curves of the local approach. Though in the AD vs. Normal experiment, the accuracy of Scale I + Scale II is not the best, and it is only $1 \%$ lower than the best one and AUC of it is 0.01 lower than the best one. Scale I + Scale II could be a good choice for the local approach. We will take it into consideration in the MCIc vs. MCInc classification.

We note that the accuracy achieved by our method is comparable to other methods in the literature. For instance, Gray et al. [18] established a method based on FDG-PET 
images with an accuracy of 88.4\%. Hinrichs et al. [21] and Zhang et al. [44] proposed methods based on the combination of multi-modality imaging and non-imaging data, and their methods achieve accuracy of $92.4 \%$ and $93.2 \%$ respectively. These results are better than ours, but their costs are much higher. For MRI only methods, Zhang et al.'s approach is $86.2 \%$ and Westman et al.'s is $87.0 \%$ [39]; Our method is in line with top results. It can be claimed that the wavelet frame features extracted from structural MRI is an appropriate approach, and we will further study the performance for the prediction of the MCInc and MCIc patients.

\section{Classification of MCI Patients}

\subsection{Classifier by $A D$ vs $N C$ data}

It is more challenging to do autodiagnostics of MCIc and MCInc patients than AD and Normal, since AD and Normal are totally different status of the disease, while MCIc and MCInc are at the similar stage of the disease. An autodiagnostics method always needs to clearly differ features of different groups, but the difference between MCIc and MCInc group seems not that significant compared to AD and NC group.

In clinical applications, there are situations that only AD and NC groups data are available. Thus we test the feasibility of using classifiers constructed by AD and NC data for classifying MCI patients. Using the two good classifiers constructed in the last section, i.e. the Hippocampus Approach Scale I-V, and Scale I+II trained by using the whole ADNI_1 screening $1.5 \mathrm{~T}$ data set, we do the classification test directly on MCI patients. Upon classing the MCInc as normal, and MCIc as AD respectively, the accuracy, specificity and sensibility are computed and shown Table 5 .

Table 5: Direct classifier

\begin{tabular}{ccccc}
\hline \multicolumn{2}{c}{ Classifier } & \multicolumn{3}{c}{ Result } \\
\hline Approach & Features & Sensitivity & Specificity & Accuracy \\
Hippocampus & Scale I+II & $70.97 \%$ & $67.61 \%$ & $69.17 \%$ \\
Hippocampus & Scale I-V & $72.58 \%$ & $66.20 \%$ & $69.17 \%$ \\
\hline
\end{tabular}

We notice that both classifiers achieve an accuracy of $69.17 \%$, and it implies that the wavelet features are still appropriate for MCIc and MCInc patients, but not as strong as the performance in the last section. It is necessary to train new classifiers using the data of MCI patients to improve the accuracy.

\subsection{Global Approach}

As in Section 13.1, a similar procedure is applied on the MCI data sets. In addition to test different levels, we also test the performance for the combined features, such as scale I+II and scale I-V. Since the number of features with all scales (156) is larger than the number of samples available (133), we discard the combination of the features with all scales to avoid over-fitting. Finally, the performance of seven classifiers trained with MCI data set is presented in Table 6. We can observe that the classifier with the combination of scale I and scale II is a good choice, since it achieves an accuracy of $71.43 \%$, sensitivity of $73.24 \%$ 
and specificity of $61.29 \%$. Figure 8 shows the ROC curves of global approach. The respective AUC of Scale I+II is 0.68 .

Table 6: Classification of MCInc vs. MCIc Using Global Features

\begin{tabular}{|c|c|c|c|c|c|c|c|c|c|}
\hline Features & Sensitivity & Specificity & $\begin{array}{c}\text { False } \\
\text { positive } \\
\text { rate }(\alpha)\end{array}$ & $\begin{array}{c}\text { False } \\
\text { negative } \\
\text { rate }(\beta)\end{array}$ & Power & $\begin{array}{c}\text { Likelihood } \\
\text { ratio } \\
\text { positive }\end{array}$ & $\begin{array}{c}\text { Likelihood } \\
\text { ratio } \\
\text { negative }\end{array}$ & Accuracy & AUC \\
\hline Scale I & $.97 \%$ & $.06 \%$ & $41.94 \%$ & $38.03 \%$ & 0.38 & 1.48 & 0.65 & $63.91 \%$ & 0.74 \\
\hline Scale II & $.20 \%$ & $58.06 \%$ & $41.94 \%$ & $33.80 \%$ & 0.34 & 1.58 & 0.58 & $66.17 \%$ & 0.71 \\
\hline Scale III & $53.52 \%$ & $33.87 \%$ & $66.13 \%$ & $46.48 \%$ & 0.46 & 0.81 & 1.37 & $48.12 \%$ & 0.53 \\
\hline Scale IV & $63.38 \%$ & $45.16 \%$ & $54.84 \%$ & $36.62 \%$ & 0.37 & 1.16 & 0.81 & $58.65 \%$ & 0.64 \\
\hline Scale V & $69.01 \%$ & $51.61 \%$ & $48.39 \%$ & $30.99 \%$ & 0.31 & 1.43 & 0.6 & $64.66 \%$ & 0.68 \\
\hline Scale VI & $59.15 \%$ & $56.45 \%$ & $43.55 \%$ & $40.85 \%$ & 0.41 & 1.36 & 0.72 & $57.89 \%$ & 0.63 \\
\hline Scale I+II & $73.24 \%$ & $61.29 \%$ & $38.71 \%$ & $26.76 \%$ & 0.27 & 1.89 & 0.44 & $71.43 \%$ & 0.69 \\
\hline
\end{tabular}

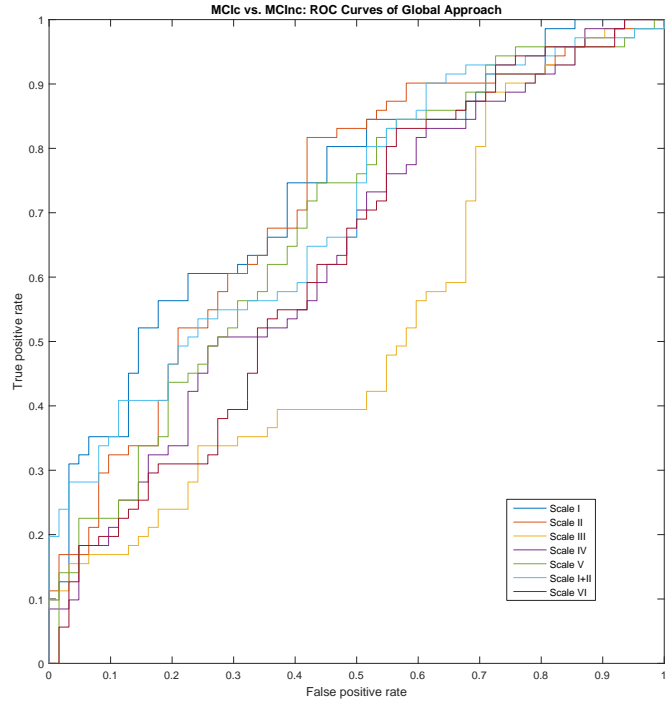

(a) Global Approach

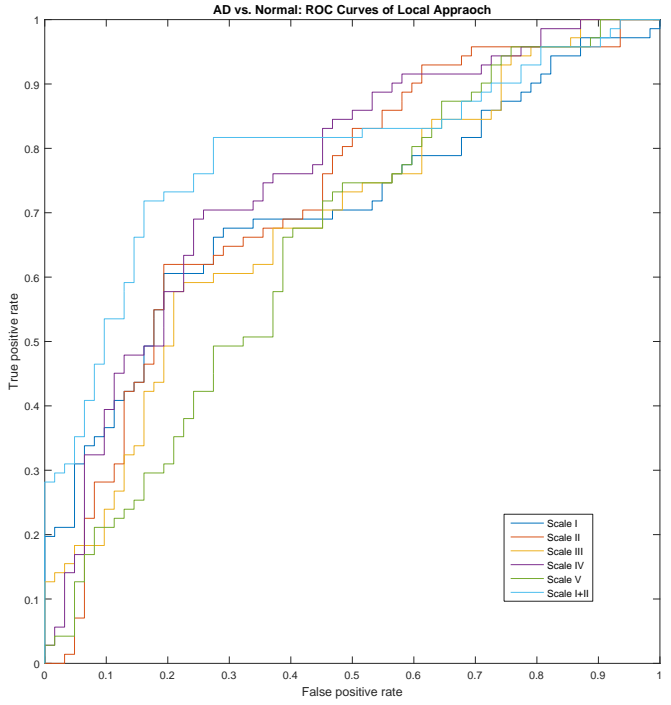

(b) Hippocampus Approach

Figure 8: ROC Curves of MCIc vs. MCInc Classifiers

\subsection{Hippocampus Approach}

We now test the performance with the classifiers constructed by the local Hippocampus sub-images of MCI data. Since the size of the subimage is smaller, we only use five level of wavelet frame decomposition. The performance of the six classifiers constructed by different feature sets are shown in Table 7 . Compared to the global approach, the features extracted from the hippocampus region provides higher accuracy. The accuracy of $76.69 \%$, sensitivity of $71.83 \%$ and specificity of $82.26 \%$ are achieved using the combined features of Scale I+II. From the ROC curves shown in Figure 8(b), we can compute that AUC is 0.72 for this classifier.

From the above results, we can see that wavelet frame coefficients could be a good choice for MCI patient classification. In the literature, researchers have attempted to use baseline 
Table 7: Classification of MCInc vs. MCIc Using Hippocampus Features

\begin{tabular}{cccccccccc}
\hline Features & Sensitivity & Specificity & $\begin{array}{c}\text { False } \\
\text { positive } \\
\text { rate }(\alpha)\end{array}$ & $\begin{array}{c}\text { False } \\
\text { negative } \\
\text { rate }(\beta)\end{array}$ & Power & $\begin{array}{c}\text { Likelihood } \\
\text { ratio } \\
\text { positive }\end{array}$ & $\begin{array}{c}\text { Likelihood } \\
\text { ratio } \\
\text { negative }\end{array}$ & Accuracy & AUC \\
\hline Scale I & $61.97 \%$ & $72.58 \%$ & $27.42 \%$ & $38.03 \%$ & 0.38 & 2.26 & 0.52 & $66.92 \%$ & 0.71 \\
Scale II & $64.79 \%$ & $67.74 \%$ & $32.26 \%$ & $35.21 \%$ & 0.35 & 2.01 & 0.52 & $66.17 \%$ & 0.73 \\
Scale III & $67.61 \%$ & $59.68 \%$ & $40.32 \%$ & $32.39 \%$ & 0.32 & 1.68 & 0.54 & $63.91 \%$ & 0.69 \\
Scale IV & $66.20 \%$ & $75.81 \%$ & $24.19 \%$ & $33.80 \%$ & 0.34 & 2.74 & 0.45 & $70.68 \%$ & 0.76 \\
Scale V & $67.61 \%$ & $56.45 \%$ & $43.55 \%$ & $32.39 \%$ & 0.32 & 1.55 & 0.57 & $62.41 \%$ & 0.65 \\
Scale I+II & $71.83 \%$ & $82.26 \%$ & $17.74 \%$ & $28.17 \%$ & 0.28 & 4.05 & 0.34 & $76.69 \%$ & 0.79 \\
\hline
\end{tabular}

measurements (imaging, cognitive, among them) to be prognostic indicator of future conversion to $\mathrm{AD}$ from MCI. The method proposed in [42] based on hippocampus longitude data can achieve the accuracy $66 \%$. The method based on FDG-PET longitude data [18] achieves $63.1 \%$. FDG-PET with more clinical information [7] achieves 76.5\%. An APOE4 stratified method achieves $76 \%$ using APOE4+, hippocampal volume and sex as features [2], while $78 \%$ for APOE- with CSF p-tau, education and sex as features. The accuracy of $76 \%$ accuracy is achived when MRI combined with PIB (Pittsburgh compund-B)-PET[34]. Wee et al. proposed a method taking the correlation of the mean cortical thickness of different regions in brain into consideration and achieves an accuracy of 75.05\% [36]; A method called deep sparse multi-task learning for feature selection has been proposed and it achieves an accuracy of $75.05 \%$ [33]. Notice that our current method only use the baseline scan images and it is much more convenient in practice, and our method can be potentially improved by combining it with more information.

Table 8 lists the comparison among several wavelet transforms with linear and Gaussian kernels. It shows that tight frame (with linear spline) features with linear kernel performs the best compared to the other methods. For the MCI patient data set, tight frame features with Gaussian kernel does not provide a satisfactory result, as we have explained in the section 2.3. Compared to other wavelet transforms, the features generated by tight frame are more efficient. Table 9 also shows the performance of polynomial kernels of different orders on tight frame features. We can see that high order polynomial kernels - for example, cubic polynomial - do not perform as well as linear kernel. In theory, the higher accuracy should be obtained for the classification of the training data when a more complex model is used. This, however, may cause over-fitting of the training data and make the generalizability of the SVM classifier low (to the testing data or any other independent data).

Table 8: Performance of features generated by several wavelet transforms with Linear or Gaussian kernel

\begin{tabular}{ccccc}
\hline Features & Kernel & Sensitivity & Specificity & Accuracy \\
\hline Gabor & Linear & $59 \%$ & $69 \%$ & $64 \%$ \\
Gabor & Gaussian & $59 \%$ & $63 \%$ & $61 \%$ \\
Haar & Linear & $63 \%$ & $61 \%$ & $62 \%$ \\
Haar & Gaussian & $66 \%$ & $63 \%$ & $65 \%$ \\
Tight Frame with Linear Spline & Linear & $72 \%$ & $82 \%$ & $77 \%$ \\
Tight Frame with Linear Spline & Gaussian & $86 \%$ & $2 \%$ & $47 \%$ \\
\hline
\end{tabular}


Table 9: Performance of Scale I+II with different polynomial kernel

\begin{tabular}{cccc}
\hline Polynomial Order & Sensitivity & Specificity & Accuracy \\
\hline Linear & $72 \%$ & $82 \%$ & $77 \%$ \\
Quadratic & $61 \%$ & $58 \%$ & $59 \%$ \\
Cubic & $58 \%$ & $50 \%$ & $54 \%$ \\
\hline
\end{tabular}

\section{Feature analysis}

In the previous two sections, the results of our method are compared to those of other methods in the literature, and the effectiveness of wavelet frame features is justified. In this section, we discuss the wavelet features from two prospective: significance of each wavelet frame feature and a survival analysis between MCIc and MCInc group. We analyze these features first by a two sample T-test, which is used to determine if the means of the two groups are equal [23], and investigate if there is significant statistical difference of the features between MCIc and MCInc patients. Furthermore, it can be found how much this difference varies with different approaches of the features generated.

Figure 9 lists the $p$-value of the two sample $t$-test of the features obtained by wavelet frames, and it contains global and local approach in AD vs Normal (and MCIc vs MCInc) diagnostics. The color of a rectangle $(i, j)$ in the graph represents the p-value of feature at Scale $i$ and Channel $j$. The features for which is significant different (with the confidence level $\alpha=0.05$ ) between MCIc vs MCInc is marked in blue. For global features, there are 31 out of 52 having significant difference in the first two scales, while for the hippocampus features, 50 features are significantly different. Moreover, each single local feature has a smaller p-value than the global feature. Thus it can be concluded that wavelet features are different in MCIc and MCInc group, and local features show even more difference than global features. It is consistent with the classification result. It is notable that the difference in AD vs Normal diagnostics is larger than that in MCIc vs MCInc, and it is consistent with the fact that the classification accuracy is higher in AD vs Normal diagnostics than the one of MCIc and MCInc. For a more quantitative comparison of difference, we compute the quantities :

$$
\begin{aligned}
\operatorname{avg}_{c}(k) & =\frac{1}{N_{c}} \sum_{i \in M C I c} x_{i}(k) \\
\operatorname{avg}_{n c}(k) & =\frac{1}{N_{n c}} \sum_{i \in M C I n c} x_{i}(k) \\
\operatorname{rel}(k) & =\frac{\operatorname{avg}_{c}(k)-\operatorname{avg}_{n c}(k)}{\operatorname{avg}_{n c}(k)}
\end{aligned}
$$

where $N_{c}$ indicates the number of MCIc samples and $N_{n c}$ indicates the number of MCInc samples. Thus $\operatorname{rel}(k)$ measures relative difference of channel $k$ in the MCIc and MCInc. We can see from Figure 10 that the relative difference of local features is larger than the global one. Both the two sample $t$ - test and the relative difference imply that hippocampus features may be more efficient than the global features, which is also implied by previous 


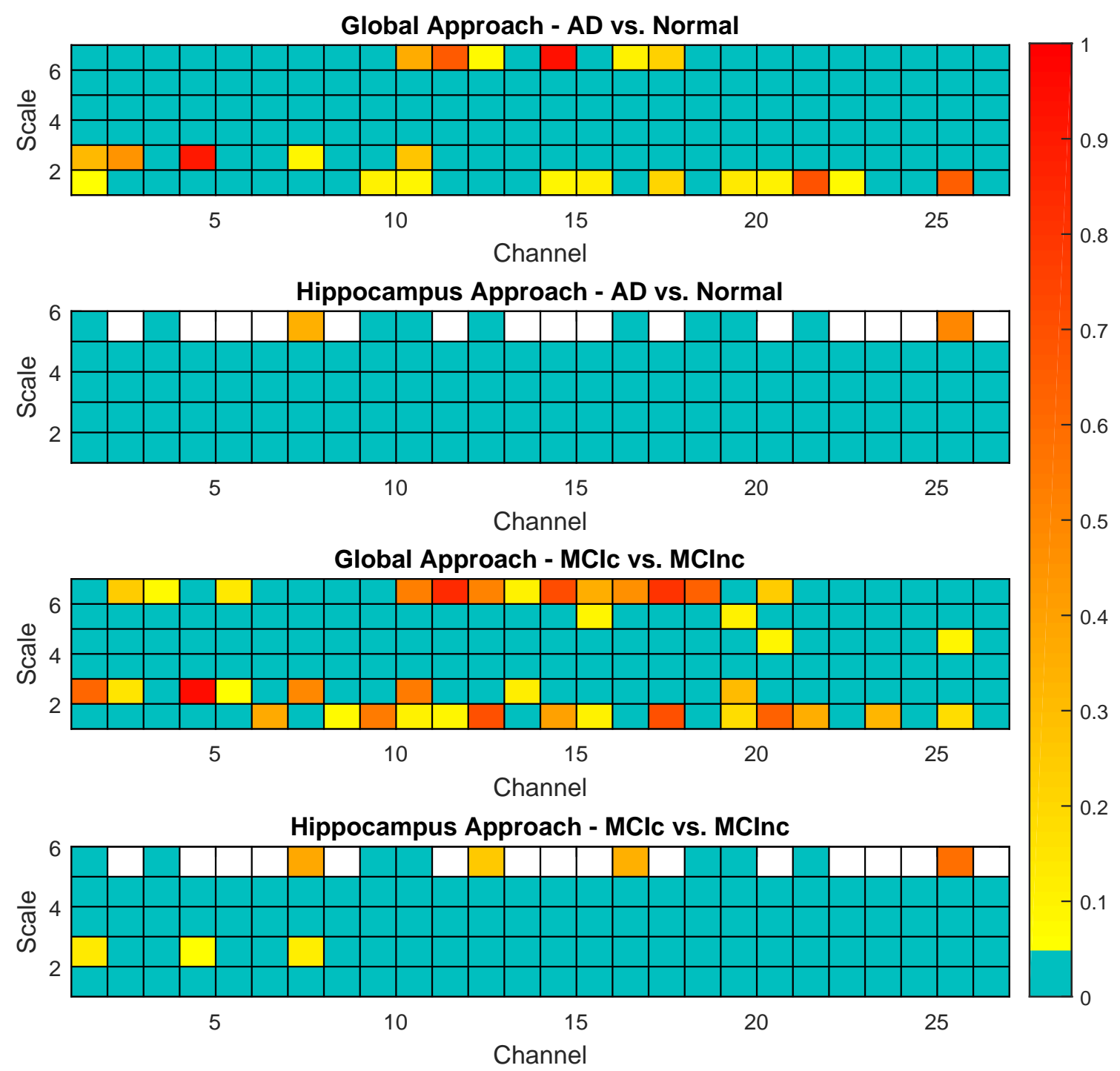

Figure 9: Two sample T-test: p-value of global and hippocampus features: The blue grids indicate that significant difference exists between the corresponding groups; the white ones indicate that the values of these channels of subjects are always 0 , therefore there is no difference; the $p$-values of remaining ones are larger than 0.05 , for which we accept the hypothesis that they are the similar. 


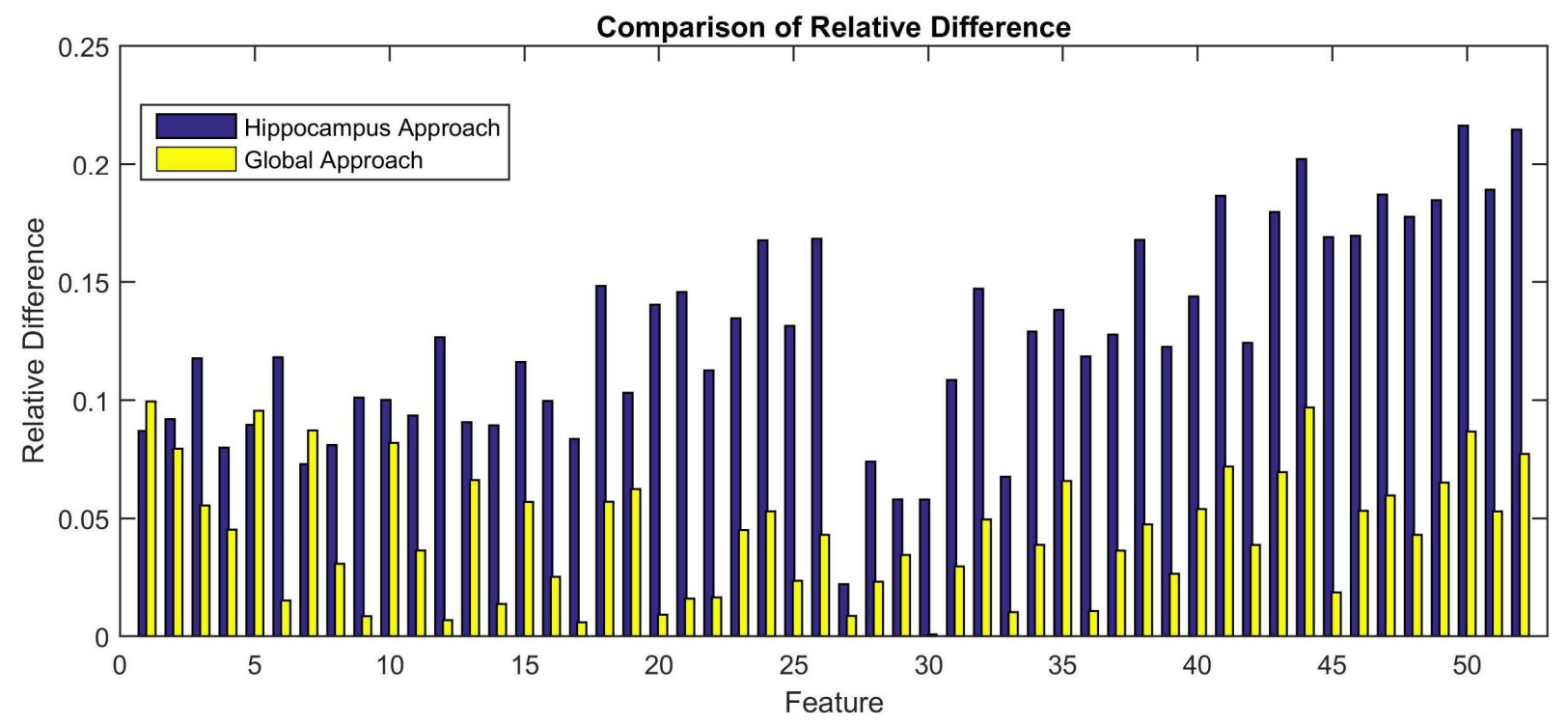

Figure 10: Relative difference of wavelet features extracted from MCIc and MCInc data by two approaches.

classification results in Section 3 and 4 , and that using hippocampus features can lead to a better classifier than the global ones both in $\mathrm{AD}$ vs $\mathrm{NC}$ and MCIc vs MCInc.

Although by the two sample $t$-test and the relevance analysis, we know that most of features are statistically efficient in diagnostics, the two analyses can not provide a quantitative measure of the influence of each feature. A Cox proportional hazard regression model [9] is introduced to analyze the survival of a patient and explanatory variables. The features of Scale I and Scale II of 71 converted MCI patients are used for the survival analysis. The Cox regression coefficients of these features after standardization are listed in Table 10. At a confidence level of 0.05 , the significant explanatory variables are marked with an asterisk. Notice that all the features are real numbers, which implies that the regression coefficient represents the increase of logarithm of hazard for the corresponding covariate value increasing 1. Thus an interpretation of the hazard ratio can be found. For example, if the value of channel 4 at scale I increases by 1, the risk of conversion to AD will increase 666.896 times. The confidential interval of channel 25 at scale I and channel 9 at scale II contain 1, thus the two features are non-influential in survival. Thus wavelet features can be potentially useful for constructing point-based tool to predict the conversion time of MCI patient [3].

\section{Conclusion}

In summary, a new AD early autodiagnostics method based on MRI data has been proposed. By extracting features using tight wavelet frames and adopting support vector machine as classifier, our method has investigated 228 normal controls and 188 AD patients from normalized data set ADNI_1 and 133 MCI patients for AD diagnosis and MCI diagnosis respectively. Accuracy for classification on AD and normal reaches $84.13 \%$ while that for MCInc and MCIc reaches $76.69 \%$ for conversion time within 36 months. And it performs best in its hippocampus approach, which is consistent with established knowledge. Compared to previous studies in the literature, our method has obtained a competitive result using the 
Table 10: Cox Regression

\begin{tabular}{|c|c|c|c|c|c|c|c|}
\hline \multirow{2}{*}{ Scale } & \multirow{2}{*}{ Channel } & \multirow{2}{*}{$\mathrm{B}$} & \multirow{2}{*}{$\mathrm{SE}$} & \multirow{2}{*}{ p-value } & \multirow{2}{*}{$\operatorname{Exp}(B)$} & \multicolumn{2}{|c|}{$95.0 \%$ CI $\operatorname{Exp}(B)$} \\
\hline & & & & & & Lower & Upper \\
\hline \multirow{26}{*}{ I } & 1 & 1.928 & 1.697 & 0.256 & 6.876 & 0.247 & 191.466 \\
\hline & 2 & -4.702 & 1.476 & $0.001 *$ & 0.009 & 0.001 & 0.164 \\
\hline & 3 & 2.79 & 1.845 & 0.13 & 16.287 & 0.438 & 606.063 \\
\hline & 4 & 6.503 & 1.342 & $0^{*}$ & 666.896 & 48.08 & 9250.255 \\
\hline & 5 & 3.831 & 1.778 & $0.031^{*}$ & 46.101 & 1.414 & 1503.407 \\
\hline & 6 & -6.841 & 2.057 & $0.001 *$ & 0.001 & 0 & 0.06 \\
\hline & 7 & -1.025 & 1.471 & 0.486 & 0.359 & 0.02 & 6.412 \\
\hline & 8 & -5.297 & 2.028 & $0.009 *$ & 0.005 & 0 & 0.267 \\
\hline & 9 & 1.481 & 1.854 & 0.425 & 4.397 & 0.116 & 166.601 \\
\hline & 10 & -7.894 & 1.837 & $0^{*}$ & 0 & 0 & 0.014 \\
\hline & 11 & -1.754 & 1.432 & 0.22 & 0.173 & 0.01 & 2.862 \\
\hline & 12 & -0.13 & 2.191 & 0.953 & 0.878 & 0.012 & 64.399 \\
\hline & 13 & 5.224 & 1.946 & $0.007^{*}$ & 185.759 & 4.101 & 8414.507 \\
\hline & 14 & -4.037 & 1.242 & $0.001^{*}$ & 0.018 & 0.002 & 0.201 \\
\hline & 15 & 5.756 & 2.559 & $0.025^{*}$ & 316.086 & 2.095 & 47685.024 \\
\hline & 16 & -4.183 & 1.594 & $0.009^{*}$ & 0.015 & 0.001 & 0.347 \\
\hline & 17 & 2.561 & 2.384 & 0.283 & 12.943 & 0.121 & 1384.287 \\
\hline & 18 & 0.243 & 3.634 & 0.947 & 1.275 & 0.001 & 1580.873 \\
\hline & 19 & 5.212 & 1.454 & $0^{*}$ & 183.484 & 10.621 & 3169.833 \\
\hline & 20 & 4.122 & 2.672 & 0.123 & 61.679 & 0.328 & 11600.691 \\
\hline & 21 & 0.915 & 2.384 & 0.701 & 2.496 & 0.023 & 267.121 \\
\hline & 22 & -9.741 & 3.702 & $0.009^{*}$ & 0 & 0 & 0.083 \\
\hline & 23 & 1.35 & 2.483 & 0.587 & 3.857 & 0.03 & 500.979 \\
\hline & 24 & -2.99 & 3.439 & 0.385 & 0.05 & 0 & 42.522 \\
\hline & 25 & 4.734 & 2.614 & 0.07 & 113.775 & 0.678 & 19101.616 \\
\hline & 26 & -4.124 & 9.535 & 0.665 & 0.016 & 0 & 2114361.1 \\
\hline \multirow{26}{*}{ II } & 1 & -4.41 & 1.144 & $0^{*}$ & 0.012 & 0.001 & 0.114 \\
\hline & 2 & 1.617 & 0.876 & 0.065 & 5.038 & 0.905 & 28.048 \\
\hline & 3 & -2.127 & 0.781 & $0.006^{*}$ & 0.119 & 0.026 & 0.551 \\
\hline & 4 & -4.775 & 1.417 & $0.001^{*}$ & 0.008 & 0.001 & 0.136 \\
\hline & 5 & -0.818 & 1.245 & 0.511 & 0.441 & 0.038 & 5.069 \\
\hline & 6 & -3.672 & 1.292 & $0.004^{*}$ & 0.025 & 0.002 & 0.32 \\
\hline & 7 & 9.762 & 2.073 & $0^{*}$ & 17356.567 & 298.633 & 1008766.239 \\
\hline & 8 & -4.929 & 1.503 & $0.001^{*}$ & 0.007 & 0 & 0.138 \\
\hline & 9 & 2.527 & 1.625 & 0.12 & 12.522 & 0.518 & 302.668 \\
\hline & 10 & 5.905 & 1.218 & $0^{*}$ & 366.88 & 33.687 & 3995.667 \\
\hline & 11 & 3.467 & 1.277 & $0.007^{*}$ & 32.036 & 2.621 & 391.642 \\
\hline & 12 & -3.084 & 1.568 & $0.049^{*}$ & 0.046 & 0.002 & 0.99 \\
\hline & 13 & -1.916 & 0.847 & $0.024^{*}$ & 0.147 & 0.028 & 0.773 \\
\hline & 14 & 13.881 & 2.896 & $0^{*}$ & 1067320.8 & 3657.672 & 311447697.7 \\
\hline & 15 & -2.108 & 2.834 & 0.457 & 0.121 & 0 & 31.382 \\
\hline & 16 & 7.906 & 1.709 & $0^{*}$ & 2713.66 & 95.21 & 77344.283 \\
\hline & 17 & -17.04 & 4.181 & $0^{*}$ & 0 & 0 & 0 \\
\hline & 18 & 4.953 & 5.949 & 0.405 & 141.558 & 0.001 & 16387284 \\
\hline & 19 & -7.249 & 1.827 & $0^{*}$ & 0.001 & 0 & 0.026 \\
\hline & 20 & -9.322 & 1.759 & $0^{*}$ & 0 & 0 & 0.003 \\
\hline & 21 & 8.197 & 1.917 & $0^{*}$ & 3628.549 & 84.718 & 155413.388 \\
\hline & 22 & 6.089 & 2.323 & $0.009 *$ & 440.945 & 4.645 & 41857.33 \\
\hline & 23 & 0.195 & 2.526 & 0.938 & 1.216 & 0.009 & 171.653 \\
\hline & 24 & 8.259 & 4.007 & $0.039 *$ & 3860.773 & 1.5 & 9936042.513 \\
\hline & 25 & -9.483 & 3.287 & $0.004^{*}$ & 0 & 0 & 0.048 \\
\hline & 26 & 3.192 & 5.717 & 0.577 & 24.332 & 0 & 1787656.4 \\
\hline
\end{tabular}


least information about the patients. The tight frame based feature contains not only boundary information but also intensity, and they are directly related to voxel information and are easy to compute compared to shape descriptor based features. To the best of our knowledge, the method developed in this work in AD early autodiagnostics is new. Meanwhile, information from other features and their integration should be useful to improve the classification performance. This important question will be investigated in future independent studies with additional tools (on top of what we studied in this paper) and larger sample sizes. For example, it may be helpful to consider a similarity map which contains the cortical thickness of different ROI and their correlations instead of the hippocampus regions, as suggested in [36]. Moreover, feature selection is a crucial technique to reduce the redundant information which is wicked for classification [33]. The features of Scale I and Scale II have show good performance and a variable selection procedure may be considered in the next step to further improve the accuracy.

\section{Acknowledgements}

Data collection and sharing for this project was funded by the Alzheimer's Disease Neuroimaging Initiative (ADNI) (National Institutes of Health Grant U01 AG024904) and DOD ADNI (Department of Defense award number W81XWH-12-2-0012). ADNI is funded by the National Institute on Aging, the National Institute of Biomedical Imaging and Bioengineering, and through generous contributions from the following: Alzheimer's Association; Alzheimer's Drug Discovery Foundation; Araclon Biotech; BioClinica, Inc.; Biogen Idec Inc.; Bristol-Myers Squibb Company; Eisai Inc.; Elan Pharmaceuticals, Inc.; Eli Lilly and Company; EuroImmun; F. Hoffmann-La Roche Ltd and its affiliated company Genentech, Inc.; Fujirebio; GE Healthcare; ; IXICO Ltd.; Janssen Alzheimer Immunotherapy Research \& Development, LLC.; Johnson \& Johnson Pharmaceutical Research \& Development LLC.; Medpace, Inc.; Merck \& Co., Inc.; Meso Scale Diagnostics, LLC.; NeuroRx Research; Neurotrack Technologies; Novartis Pharmaceuticals Corporation; Pfizer Inc.; Piramal Imaging; Servier; Synarc Inc.; and Takeda Pharmaceutical Company. The Canadian Institutes of Health Research is providing funds to support ADNI clinical sites in Canada. Private sector contributions are facilitated by the Foundation for the National Institutes of Health (www.fnih.org). The grantee organization is the Northern Rev December 5, 2013 California Institute for Research and Education, and the study is coordinated by the Alzheimer's Disease Cooperative Study at the University of California, San Diego. ADNI data are disseminated by the Laboratory for Neuro Imaging at the University of Southern California.

The authors would like to thank the anonymous reviewers for their helpful comments in improving the presentation of this paper. The work of the authors were partially supported by NSFC 91330102 and 973 program (2015CB856000).

\section{References}

[1] Y. Aksu, D. J. Miller, G. Kesidis, D. C. Bigler, and Q. X. Yang. An MRI-derived definition of MCI-to-AD conversion for long-term, automatic prognosis of MCI patients. PLoS ONE, 6(10):e25074, 2011. 
[2] L. G. Apostolova, K. S. Hwang, O. Kohannim, D. Avila, D. Elashoff, C. R. J. Jr., L. Shaw, J. Q. Trojanowski, M. W. Weiner, and P. M. Thompson. Apoe4 effects on automated diagnostic classifiers for mild cognitive impairment and Alzheimer's disease. NeuroImage: Clinical, 4(0):461 - 472, 2014.

[3] D. E. Barnes, I. S. Cenzer, K. Yaffe, C. S. Ritchie, S. J. Lee, Alzheimer's Disease Neuroimaging Initiative, et al. A point-based tool to predict conversion from mild cognitive impairment to probable Alzheimer's disease. Alzheimer's \& Dementia, 10(6):646-655, 2014.

[4] D. S. Beal, V. L. Gracco, J. Brettschneider, R. M. Kroll, and L. F. D. Nil. A voxel-based morphometry (VBM) analysis of regional grey and white matter volume abnormalities within the speech production network of children who stutter. Cortex, 49(8):2151 2161,2013 .

[5] A. Chincarini, P. Bosco, P. Calvini, G. Gemme, M. Esposito, C. Olivieri, L. Rei, S. Squarcia, G. Rodriguez, R. Bellotti, et al. Local MRI analysis approach in the diagnosis of early and prodromal Alzheimer's disease. NeuroImage, 58(2):469-480, 2011.

[6] M. Chupin, E. Gérardin, R. Cuingnet, C. Boutet, L. Lemieux, S. Lehéricy, H. Benali, L. Garnero, and O. Colliot. Fully automatic hippocampus segmentation and classification in Alzheimer's disease and mild cognitive impairment applied on data from ADNI. Hippocampus, 19(6):579-587, 2009.

[7] D. G. Clark. Residual vectors for Alzheimer disease diagnosis and prognostication. Brain and Behavior, 1(2):142-152, 2011.

[8] C. Cortes and V. Vapnik. Support-vector networks. Machine Learning, 20(3):273-297, 1995.

[9] D. R. Cox. Regression models and life-tables. Journal of the Royal Statistical Society. Series B (Methodological), 34(2):187-220, 1972.

[10] I. Daubechies. Ten Lectures on Wavelets. CBMS-NSF Lecture Notes,. SIAM, 1 edition, 1992.

[11] B. Dong and Z. Shen. MRA-based wavelet frames and applications, volume 19 of IAS Lecture Note Series. Park City Mathematics Institute, 2010.

[12] D. Donoho, S. Mallat, R. von Sachs, et al. Estimating covariances of locally stationary processes: rates of convergence of best basis methods. Statistics, Stanford University, Standford, California, USA, Tech. Rep, 1998.

[13] R.-E. Fan, P.-H. Chen, and C.-J. Lin. Working set selection using second order information for training support vector machines. The Journal of Machine Learning Research, 6:1889-1918, 2005.

[14] T. Fawcett. Roc graphs: Notes and practical considerations for researchers. Machine Learning, 31:1-38, 2004. 
[15] R. Filipovych and C. Davatzikos. Semi-supervised pattern classification of medical images: Application to mild cognitive impairment (MCI). NeuroImage, 55(3):1109 - 1119, 2011.

[16] R. A. Gopinath and H. Guo. Introduction to wavelets and wavelet transforms: a primer, 1997.

[17] M. Goto, O. Abe, S. Aoki, N. Hayashi, T. Miyati, H. Takao, T. Iwatsubo, F. Yamashita, H. Matsuda, H. Mori, A. Kunimatsu, K. Ino, K. Yano, and K. Ohtomo. Diffeomorphic anatomical registration through exponentiated lie algebra provides reduced effect of scanner for cortex volumetry with atlas-based method in healthy subjects. Neuroradiology, 55(7):869-875, 2013.

[18] K. R. Gray, R. Wolz, R. A. Heckemann, P. Aljabar, A. Hammers, D. Rueckert, Alzheimer's Disease Neuroimaging Initiative, et al. Multi-region analysis of longitudinal FDG-PET for the classification of Alzheimer's disease. NeuroImage, 60(1):221-229, 2012.

[19] M. Grundman, R. C. Petersen, S. H. Ferris, R. G. Thomas, P. S. Aisen, D. A. Bennett, N. L. Foster, C. R. Jack Jr, D. R. Galasko, R. Doody, et al. Mild cognitive impairment can be distinguished from Alzheimer disease and normal aging for clinical trials. Archives of neurology, 61(1):59-66, 2004.

[20] K. Hackmack, F. Paul, M. Weygandt, C. Allefeld, and J.-D. Haynes. Multi-scale classification of disease using structural MRI and wavelet transform. NeuroImage, 62(1):48 $58,2012$.

[21] C. Hinrichs, V. Singh, G. Xu, and S. C. Johnson. Predictive markers for AD in a multi-modality framework: An analysis of MCI progression in the ADNI population. NeuroImage, 55(2):574 - 589, 2011.

[22] C.-W. Hsu, C.-C. Chang, C.-J. Lin, et al. A practical guide to support vector classification, 2003.

[23] D. H. Jones. Journal of Educational and Behavioral Statistics, 19(3):pp. 304-307, 1994.

[24] H. Liu, R. H. Chan, and Y. Yao. Geometric tight frame based stylometry for art authentication of van gogh paintings. CoRR, abs/1407.0439, 2014.

[25] S. Mallat. A wavelet tour of signal processing. Academic press, 2nd edition, 1999.

[26] Y. Mu and F. H. Gage. Adult hippocampal neurogenesis and its role in Alzheimer's disease. Molecular Neurodegeneration, 6(1):85, 2011.

[27] S. G. Mueller, M. W. Weiner, L. J. Thal, R. C. Petersen, C. Jack, W. Jagust, J. Q. Trojanowski, A. W. Toga, and L. Beckett. The Alzheimer's disease neuroimaging initiative. Neuroimaging Clinics of North America, 15(4):869 - 877, 2005. Alzheimer's Disease: 100 Years of Progress. 
[28] K. Nakazawa, T. J. McHugh, M. A. Wilson, and S. Tonegawa. Nmda receptors, place cells and hippocampal spatial memory. Nature Reviews Neuroscience, 5(5):361-372, 2004.

[29] J. Platt et al. Probabilistic outputs for support vector machines and comparisons to regularized likelihood methods. Advances in large margin classifiers, 10(3):61-74, 1999.

[30] M. Prince, M. Guerchet, M. Prina, B. U. P. Association, et al. World Alzheimer Report 2013: Journey of Caring: An analysis of long-term care for dementia. Alzheimer's Disease International, 2013.

[31] F. Provost and T. Fawcett. Robust classification for imprecise environments. Machine Learning, 42(3):203-231, 2001.

[32] A. Ron and Z. Shen. Affine systems in $L_{2}\left(R^{d}\right)$ : the analysis of the analysis operator. Journal of Functional Analysis, 148:408-447, 1997.

[33] H.-I. Suk, S.-W. Lee, D. Shen, A. D. N. Initiative, et al. Deep sparse multi-task learning for feature selection in alzheimers disease diagnosis. Brain Structure and Function, pages $1-19,2015$.

[34] P. T. Trzepacz, P. Yu, J. Sun, K. Schuh, M. Case, M. M. Witte, H. Hochstetler, and A. Hake. Comparison of neuroimaging modalities for the prediction of conversion from mild cognitive impairment to Alzheimer's dementia. Neurobiology of Aging, 35(1):143 $151,2014$.

[35] N. Tzourio-Mazoyer, B. Landeau, D. Papathanassiou, F. Crivello, O. Etard, N. Delcroix, B. Mazoyer, and M. Joliot. Automated anatomical labeling of activations in spm using a macroscopic anatomical parcellation of the mni mri single-subject brain. Neuroimage, 15(1):273-289, 2002.

[36] C.-Y. Wee, P.-T. Yap, D. Shen, and for the Alzheimer's Disease Neuroimaging Initiative. Prediction of alzheimer's disease and mild cognitive impairment using cortical morphological patterns. Human Brain Mapping, 34(12):3411-3425, 2013.

[37] M. W. Weiner, P. S. Aisen, C. R. J. Jr., W. J. Jagust, J. Q. Trojanowski, L. Shaw, A. J. Saykin, J. C. Morris, N. Cairns, L. A. Beckett, A. Toga, R. Green, S. Walter, H. Soares, P. Snyder, E. Siemers, W. Potter, P. E. Cole, and M. Schmidt. The Alzheimer's disease neuroimaging initiative: Progress report and future plans. Alzheimer's 85 Dementia, 6(3):202 - 211.e7, 2010.

[38] M. W. Weiner, D. P. Veitch, P. S. Aisen, L. A. Beckett, N. J. Cairns, R. C. Green, D. Harvey, C. R. Jack, W. Jagust, E. Liu, et al. The Alzheimers disease neuroimaging initiative: A review of papers published since its inception. Alzheimer's $\&$ Dementia, 8(1):S1-S68, 2012.

[39] E. Westman, J.-S. Muehlboeck, and A. Simmons. Combining MRI and CSF measures for classification of Alzheimer's disease and prediction of mild cognitive impairment conversion. NeuroImage, 62(1):229 - 238, 2012. 
[40] A. Wimo and M. J. Prince. World Alzheimer Report 2010: the global economic impact of dementia. Alzheimer's Disease International, 2010.

[41] R. Wolz, P. Aljabar, J. V. Hajnal, A. Hammers, D. Rueckert, Alzheimer's Disease Neuroimaging Initiative, et al. Leap: learning embeddings for atlas propagation. NeuroImage, 49(2):1316-1325, 2010.

[42] R. Wolz, R. A. Heckemann, P. Aljabar, J. V. Hajnal, A. Hammers, J. Ltjnen, and D. Rueckert. Measurement of hippocampal atrophy using 4d graph-cut segmentation: Application to ADNI. NeuroImage, 52(1):109 - 118, 2010.

[43] B. T. Wyman, D. J. Harvey, K. Crawford, M. A. Bernstein, O. Carmichael, P. E. Cole, P. K. Crane, C. DeCarli, N. C. Fox, J. L. Gunter, et al. Standardization of analysis sets for reporting results from ADNI MRI data. Alzheimer's \& Dementia, 9(3):332-337, 2013.

[44] D. Zhang, Y. Wang, L. Zhou, H. Yuan, D. Shen, Alzheimer's Disease Neuroimaging Initiative, et al. Multimodal classification of Alzheimer's disease and mild cognitive impairment. NeuroImage, 55(3):856-867, 2011. 